\section{A CASE OF ADHESION OF THE LABIA,}

BY WHICH THE BIRTH OF A CHILD WAS IMPEDED FOR AN HOUR; DIVISION OF THE PARTS FOLLOWED BY IMMEDIATE EXPULSION OF THE INFANT.

By JAMES REID, Esa.,

QURGEON TO THE KENT AND CAXTRBBURY HOSPITAL, CAMBBIDGE.

(Read at the meeting of the Cambridge and Huntingdon Branch Associatiox.)

WITH REMARKS ON ATRESIA VAGIN A AND SIMILAR
MALFORMATIONS.

BY GEORGE MURRAY HUMPHRY, Esa., BUBGBOX TO ADDENBROOKE'S HOSPITAY.

ON the 6th of May, 1850, I was requested by Mr. C. Holtum, a surgeon in this town, to visit a patient of his, who was in labour of her first child. Labour had commenced in the morning, and Mr. Holtum had ascertained, without encountering any obstruction in passing his finger into the vagina, that the presentation was natural. By six o'clock in the evening the head had descended into the pelvis, and was pressed forwards by each pain to the perineum; Mr. Holtum was then so satisfied, that labour would soon terminate, that he assured the patient that it would be all over, and he should be able to leave the house, in half an hour. The head passed on to the perineum, and had distended it very forcibly, when it became evident that the soft parts offered a stronger resistance than usual to the passage of the child. An examination was made during the interval of a pain. The labia were found to be completely united from their superior angle down to the orifice of the vagina; they were quite natural, and were expanded over the head of the child in a uniformly even surface that extended to the mons veneris. There was no groove or depression between them, but the course of their union was marked by a line, which was distinguished by the paler colour of the integument. The os externum was a complete circle, of an inch and a half diameter, and had pressed against the head so as to form a corresponding tumefaction on the scalp. The perineum was soft and natural, and was distended by the child's head, which had almost passed the bony outline of the lower aperture of the pelvis. A strong expulsive effort occurred, and it was seen that the orifice did not expand, but that the whole integuments of the perineum and of the external parts were pushed forwards, and distended to such a degree that it was quite evident laceration would take place before the child could be expelled, and, in all probability, that the rupture would take place in the direction of the perineum, which covered the greater part of the head, and was more immediately exposed to the direction of the moving power. A director and bistoury were obtained, and the integuments were divided along the central pale line towards the pubes, the thickness of the divided parts increasing in that direction from the one-eighth of an inch to half an inch. There was scarcely any bleeding. A pain immediately occurred, and a living child was expelled. Lint was inserted to prevent adhesion. The progress of the labour was delayed, at least, an hour by the cohesion of the labia. One could scarcely have expected, beforehand, that such a cause would have formed so great an obstacle. The remedy was obvious and simple, and the result immediate. The patient subsequently did well.

From the complete state of the union between the labia, from the absence of any traces of ulceration, in fact, from the otherwise natural condition of the parts, $I$ was led to infer that the cohesion was congenital, and the history the patient subsequently gave upon the subject. confirmed the conclusion.

She was 30 years of age, and had been twice married. She had never known that there was anything wrong or unusual in the organs of generation. She always menstruated freely, and had no difficulty in making water, the urine issuing in a stream; the obliquo direction, downwards and forwards, of the stream would perhaps explain this. In her first connection with her former husband she said that there was no more obstruction than what she thought was usual. He lived about five years, but she was never pregnant during that period. He had led a gay life before he married her, and died of consumption. She had been married to her second husband about twelve months, and he had not met with any difficulty. The opening in the external parts opposite the vagina was, therefore, sufficient for the complete act of coition.

Numerous cases are on record of obstructed labour from contraction of the vagina, from complete or partial occlusion of its calibre by horizontal or vertical bands, by thickened membranes and adhesions of surfaces, placed cither at the orifice or deeper, and which have been caused either by cicatrices formed after ulcerations, wounds, and lacerations after difficult labours, or have, in some instances, had an origin in utero. From all these, the obstruction in the present case should be distinguished, inasmuch as, it was from adhesion of parts external to the vagina that the obstacle to the birth of the child existed, and thero was every reason to believe that the vagina itself was in a natural state.

Adhesion of the labia as a congenital affection, or following excoriations, is mentioned by many authors, and in most works on jurisprudence, but the instances recorded in which it has formed an obstacle in labour appear to be few; this, no doubt, is in great measure to be explained by the defect having been discovered and remedied beforehand. It might be further explained by the supposition that females in whom it exists to any great degree are impotent, but it appears that so long as there is the smallest opening communicating with the vagina, and other circumstances are favourable, pregnancy may take place. At present I have only been able to find two cases, about one of which there is a doubt whether it does not belong more properly to the class of cases first alluded to. In a third case, pregnancy had taken place, but the evil was remedied before labour occurred. One of these caseis 
is so similar to the present one, and furnishes an example of such a great extent of adhesion, that I may perhaps be excused for giving a short abstract of it ; it is related in Dr. Merriman's work on "Difficult Parturition," p. 230. Fourth edition.

Dr. Tucker, of Malton, in September, 1781, was requested to attend a patient in labour. Upon examination the labia pudendi were observed to have the usual situation and appearance, but being expanded they were found to be connected by a strong thick opaque membrane of neaily a finger's breadth, and not distinguishable from their external skin in texture and appearance. The membrane was in front of the usual situation of the hymen, and was stretched from the surface of the perineum, of which it seemed a continuation or production, over the longitudinal sulcus between the labia, covering the mouth of the vagina, the orifice of the urethra and the clitoris, and obliterating the angles at which the labia meet above and below. Near the middle of the sulcus, there was a circular aperture, just large enough to admit a female catheter of an eighth of an inch diameter; this opening was placed just above the orifice of the urethra. The membrane resisted the forcible impulse of the child's head during several pains ; it was divided by scissors; scarcely a drop of blood followed, and the child was born.

The second case, perhaps, included more than adhesion of the external parts of generation, and is, therefore, not strictly a parallel case. It is related by $\mathbf{M r}$. Ogden in " Clay's Obstetric Record," Vol. I., 1848, p. 34 ; and is quoted in " Braithwaite's Retrospect," Vol. XXVII., Art. 135. In this case neither labia, nymphæ, clitoris, or vagina, could be found, but only the orifice of a urethra, and a flat hard tumour like the foetal head, covered by integument. By an incision of an inch and a half in depth between the meatus urinarius and the rectum, the vagina and membranes were penetrated, and in an hour a still-born child was expelled. The patient had been previously operated upon for retained menses, when an opening had been apparently made into the vagina through the urethra, by which the menses escaped, and through which impregnation had been effected.

In a case mentioned in Vol. XI. of the "MedicoChirurgical Transactions," a negro woman, ałout 27 years of age, who was in an advanced state of pregnancy, declared she could not be delivered, as there was no passage. She was examined, and a cicatrix was discovered extending from the mons veneris to within an inch of the anus, where there existed a small opening which admitted a small female catheter. According to the woman's statement, the cicatrix was the consequence of an operation performed in her native country to preserve her chastity. The adhesion was divided, and delivery accomplished. Other cases are mentioned in which the defect was noticed and remedied before pregnancy took place. One is related in Vol. XXXVII. of the Edinburgh Medical and Surgical Journal, p. 26. A small round opening, the size of a pin's head, near the urethra, was the only orifice found.
When the membrane wras divided the vagina was discovered to be quite natural. Dr. Ryan, in his "Manual of Jurisprudence," mentions that he has seen four cases of adhesion of the labia in the adult so complete, that only a small probe could be introduced at the superior commissure. Mr. W. P. Ormerod has kindly favoured me with the particulars of a case which he remembers to have met with. "A girl about 12 or 13 years of age had the labia firmly united by a thick adhesion of a substantial kind, and closing a great part of the opening of the vagina. The girl was in good health, and presented no marked traces of ulceration that he could remember, but the mother attributed the adbesion to the effect of ulceration or some disease of the parts during childhood. The adhesion was divided, and appeared to be simple union, without anything otherwise wrong or wanting about the parts ; there was, however, great difficulty in keeping the parts separate, and also in restraining the patient while the parts were divided, so that the separation of the parts even after healing was imperfect."

The existence of such an adhesion in adults is very rare, compared with the frequency of the occurrence in children. Many authors mention the frequency of the mal-conformation at the latter age, and most practitioners must have had children brought to them with the labia more or less united. Denman, who observed, and was the first apparently to mention, the frequency of the complaint at one age, and the rarity at the other, was led to remark that it was probable that the constant and free use of the limbs when the child begins to walk caused a separation without any other assistance. That the cohesion is detected and remedied in childhood, and rarely remains undiscovered till adult age, or that some accident ruptures the union, seem to be more probable explanations ; for the natural movement of the limbs produce little or no separative pressure upon the labia.

The knowledge of the serious consequences that may arise from this cohesion of the labia, and the ease with which, by mere pressure sometimes, it is destroyed in children, should perhaps lead the accoucheur, in his examination of the cbild after birth, to look more attentively to these parts, and at once interfere. Evanson and Maunsell, however, in the fourth edition of their work, page 201 , in reference to these adhesions, says :- "Nothing is required or proper to be done during infancy, unless the opening of the urethra is interfered with."

Nearly all who allude to the existence of the adhesion in children attribute it in great measure to the effects of excoriations. Without intending to deny such a result, I suspect the adhesion from excoriation is the exception, and that generally it is a congenital affection. Mr. Lawrence speaks of the adhesion as a congenital mal-conformation only, and this seems to be the case generally for the following reasons:-

There is no doubt, that the connection between the parts is often found existing at birth.

The existence of excoriation in many instances is inferred only from the presence of the adhesion. 
Adhesion of the labia is by no means constantly or frequently found in dirty children, and those likely to be excoriated.

Excoriations are commonly observed to take place in children without adhesion following, as from gonorrhœes.

\section{Mr. Humphry's Remarks on Atresia Vagine and} similar Malformations.

We may safely coincide with Mr. Reid in his opinion respecting the nature of the cohesion between the labia in the above related cases, and in others of the like kind. His arguments, added to the description given of the nature of the cohesion, and the analogy afforded by the occlusion of other orifices, are, I think, quite sufficient to establish the fact of its being a congenital malformation; indeed it can scarcely be otherwise. But a doubt may arise as to the mode in which such a malformation is likely to be produced. On this point two views may be entertained. The deformity may be supposed to result, either from an excess of development, whereby a superfluons structure is produced, or from the imperfect removal of some fœetal tissue, in consequence of which a structure, intended only to be temporary, has become persistent. These two causes, seemingly different, are in truth nearly allied, so that it is not easy to make a nice distinction between them; probably they both combine, in a greater or less degree, to produce the variety in question. The observations I have to make are chiefly in reference to the latter of these two causes, because $I$ think that it does not receive quite sufficient attention as a source of malformation.

In the formation of the fotus, and in the evolution of its several organs and tissues, it is evident that there must be two processes in continual operation; by one of these the embryonic or temporary structures are gradually transformed into the new and permanent tissues; by the other they are removed from parts where the permanent tissues are not intended to exist. The effective continuance of both these processes is essential to the formation of the proper shape and structure of the body, and of each individual part of it. If the former fail there will be an imperfection of structure combined, probably, with an imperfection of shape; if the latter fail there will be a malformation; a superabundance of structure, a junction of parts which should be separate, a closure of canals which should be open.

It is probable that these two processes, by which the several layers and structures of the embryo acquire their distinctive characters and become separate from one another, are cotemporaneous, or nearly so. One of the earliest changes observed in the germinal membrane-or the membrane in immediate contact with the yolk-is its separation into two layers,--the " serous" and the "mucous." From the former or outermost of these, the external or animal parts, and from the mucous or innermost, the internal or organic parts of the foetus are evolved. As the structure of these two layers becomes more distinct, so does their separation become more complete, till they are disunited in nearly their whole extent, and a cavity is formed between them, possessing an epithelial lining. This cavity is divided by the diaphragm into its pleural and peritoneal compartments. The mucous and serous layers of the germinal membrane remain united in the middle line, where a third or vascular layer is formed between them, in which the heart and great vessels are evolved; the large arterial and venous trunks, the representatives of this middle layer of the germinal membrane, remaining through life the great bond of union between the organic and the animal divisions of the body, which are formed respectively from the mucous and serous layers above mentioned. It is evident, therefore, that the visceral and parietal layers of the peritoneum and pleura were originally united in the germinal membrane, their separation taking place, probably, at the same time with the evolution of their structure.

In the further progress of development the outer or serous layer of the germinal membrane, which grows more rapidly than the inner or mucous, becomes bent inwards upon the yolk, whereby the embryo assumes a boat-like shape, is raised upon the surface of the yolk, and is "pinched off" from it. The sides of this outer layer, which form the walls of the thorax and abdomen, tend to the middle line in front, and meet there, cutting off the inner part of the embryo from the yolk, except at the naval, where a tubular process-the omphalomesenteric duct, with its vessels-still forms the medium of communicatlon between the intestinal canal and the yolk sac. The latter subsequently dwindles away, the omphalo-mesenteric duct and the vessels at the same time disappearing. The inflexion of the outer layer of the germinal membrane takes place at the upper or cranial, and at the lower or caudal, extremity of the embryo, as well as at the sides, forming a thick rounded end in either direction. The embryo may now be said to consist of an oval sac, formed by the serous layer of the germinal membrane enclosing an inner smaller sac, formed by the mucous layer. The mucous sac has no external communication, except with the yolk, or umbilical vesicle as this part of the yolk is now called, through the omphalo-mesenteric duct which is soon obliterated; it is entirely closed in and covered by the external and larger sac. It forms the intestine, and from it the other alimentary organs, as well as the respiratory and urinary apparatus are developed as diverticula. At first it is a good deal shorter than the external sac, but it gradually elongates, and fissures are soon formed through the wall of the external sac into its cavity, so as to lay it open at the two ends of the embryo. The anterior orifice commences on the surface of the embryo, as a depression, which extending inwards meets the superior blind extremity of the mucous, or intestinal sac, and communicating with it forms the oral aperture (not strictly the mouth) for the alimentary and respiratory organs. In like manner a depression at the lower end of the embryo meets and communicates with the inferior blind extremity of the mucous sac, constituting the cloaca or common opening of the alimentary, urinary, and genital apparatus. Both these openings must be formed by a removal of 
the substance intervening between the interior of the mucous sac and the surface of the embryo.

A still more remarkable process takes place in connection with these openings, between the second and third months. The oral orifice consists at present only of a great hiatus between the upper branchial arches. The mouth is not yet formed. First, the upper and lower jaws are developed from the superior branchial arch; then the lips are produced from the margins of the jaws, above and below, and approaching one another, coalesce so as to form a plane surface of rudimentary structure quite closing the mouth. Subsequently this rudimentary structure becomes developed into the several tissues that compose the lips, except in the line corresponding with the future opening of the mouth; in this latter situation absorption takes place instead of further development, the rudimentary structure is removed, and the true orifice is formed.* Supposing this process of absorption to fail, the rudimentary structure would become persistent and the lips would remain coherent, giving rise to the deformity, which has been described under the name artresia oris. The eyelids, in like manner, growing from the margins of the orbit cover the eyeball, and meeting over it, are united together, becoming separated by the absorption of the fotal tissue at the line of union when the several structures peculiar to the lids are developed in it above and below. $\dagger$ In puppies and kittens this separation does not take place till some days after birth; and instances are recorded of the uniting medium remaining persistent in man. The condition so engendered is called ancyloblepharon. $\neq$ The nostrils also are, after the ninth week, closed, as with a plug, by a membrane of the same nature as that closing the mouth and eyelids. $\$$ When this membrane is not removed the condition called atresia narium results.

Forasmuch as the early mode of formation of the cloaca at the lower extremity of the fotus is similar to that of the primitive oral aperture, it seems a reasonable inference that, in the further process of development, when the perineum is formed, the vagina is, like the mouth, nose, and eyes, closed by a rudimentary struc. ture, and that, as the labia acquire their proper structure, so the rudimentary tissue which had united them is removed and the opening is established. Some such process is hinted at by Bischoff, but I have been unable to find any clear account of it in the descriptions of the development of these parts, given by Valentine, Wagner, Baer, or other writers upon the subject. The persistence of this rudimentary structure, constituting atresia vagina, would accord exactly with the condition we so often see in young children, and with that occasionally observed, in the adult, for instance in Mr. Reid's case. As a general rule it would appear that the removal of this structure takes place from above downwards, for in little children who are the subjects of cohesion of the labia there is generally an orifice at the upper part

* Bischoff, entwickelungsgeschichte. Leipsig, 1842. p. 300 .

+ Bischoff, l.c. p. 227. Wagner, Elements of Physiology by Willis. p.176

\$ Baer, Lehre von dem Augenkrankheitem, rol. ii., p. 123.

Bischoff, l. c., p. 234. near to the opening of the urethra, and a probe may be passed through this orifice into the vagina behind the uniting medium, which in every case that I have seen, has been thin and soft, so that slight pressure with the probe was sufficient to divide it and bring the parts to their natural state. Neither have I ever known more than a few drops of blood to be lost or any tendency to the reunion of the labia after this slight and simple operation. In Mr. Reid's case it appears that the opening took place through the lower part of the membrane, the labia remaining united above; and in the case quoted by him from Merriman, the opening was in the middle, the labia coherent above and below.

It would seem that at a certain period of intra-uterine life the immediate orifice of the vagina proper is also closed by a second membrane uniting the crura of the clitoris. The persistent remains of the margin of this membrane probably constitutes the "hymen;" occasionally the entire membrane is persistent, causing occlusion of the vagina and retention of the menses. The imperforate condition of the vagina described by Mr. Mayo, in this journal, page 538, and the imperforate anus would appear to depend upon the imperfect formation of the cloaca, the depression or fissure produced on the external surface of the embryo not coalescing with the internal mucous or intestinal pouch, rather than upon the persistence of a rudimentary structure subsequently developed.

A well-known example of a rudimentary structure, analogous to those $I$ have been describing, is furnished by the membrana pupillaris which is almost invariably absorbed when the iris has acquired its proper structure. This occurrence takes place a short time previous or subsequent to birth. Dr. Jacob* says, that in every instance where he has made the examination, he has found the membrana pupillaris existing in a greater or less degree of perfection in the new-born infant; frequently it was perfect without the smallest breach, sometimes it presented ragged apertures in several places; and in other instances, nothing existed but a remnant hanging across the pupil like a cobweb. Considering the late period at which the membrana pupillaris is removed, it is remarkable that it should so rarely be persistent. Mr. Lawrencet observes, that so far as his researches have gone, there is no evidence of the membrana pupillaris having ever been found perfect even a few days after birth; and he believes that no record exists of any case in which an operation has been performed upon it.

Nearly related also to the subject we are upon is the formation and subsequent removal of those structures and organs which are produced to serve only a temporary purpose in the economy of the fotus or the child. Such are the allantois, the Wolffian bodies, the omphalomesenteric duct and vessels, the thymus gland, the umbilical arteries and veins, the ductus arteriosus and venosus, and the eustachian valve. Of these it may be remarked as a general rule that, when their function

- Medico-Chirurgical Transactions, Vol. xii.

+ Lawrence on Diseases of the Bye, p. 444. 
terminates before birth their structure is entirely removed, when their function is continued up to birth some traces, at any rate, of their structure are persistent through life. The Wolffian bodies and the omphalo-mesenteric duct, for instance, have entirely disappeared at birth; except in a few cases where the remains of the latter are preserved, in the form of a diverticulum, connected with the lower end of the ilium, the omphalo-mesenteric vessels being also, in such cases, sometimes represented by a thick cord passing from the diverticulum to the mesentery.

It is a fact of some practical importance that when a fotal structure is persistent it is endued with the same growing properties as the rest of the body. This is true of those fotal structures which are always persistent; for instance, the remains of the umbilical arteries and veins not only increase in length, but acquire also considerable thickness, and form strong tough cords of fibrous tissue. It is true also of those structures which are occasionally persistent: thus a diverticulum often equals the intestine in size, and the remains of the omphalo-mesenteric vessels when they are persistent form a thick, strong, fibrous cord, which bas been known to strangulate large portions of bowel entangled in the loop between it and the mesentery. There is a specimen of this sort in the museum of the College of Surgeons, and there is another, probably of the same kind, in the pathological museum belonging to the University of Cambridge. The membrane uniting the labia, which in the little child is so thin and soft that it easily yields to the pressure of a probe, is in the adult so thick and strong as to withstand the expulsive force of the uterus, and to require a scalpel for its division. In this respect, as well as in some other particulars, these structures bear much resemblance to cicatrix.

Some three or four years ago, in dissecting a fotus of about six months, I found the costal and pulmonary pleuræ on both sides of the chest united by a soft substance, nearly resembling fine areolar tissue. I have ohserved the same thing occasionally in very young children; and it is no unusual circumstance in older persons to find one or both pleuræ, and even the pericardium, universally adherent, without our being able to discover that any symptoms of pleurisy or pericarditis had been observed during life. May it not be that in some of these cases the adhesions are dependent on an imperfect separation of the membranes in the early stages of fotal life, or the persistence of some perimordial substance which should have been removed, and its development into a tissue of low kind, similar to those we are in the habit of recognising as the product of inflammation. This is but a suggestion, the value of which must be tested by more extensive observation.
PRACTICAL

\section{OBSERVATIONS ON DELIRIUM.}

\author{
By THOMAS SALTER, F.L.S., F.R.C.S.,
}

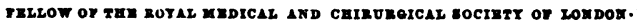

(Road before the Dorsetshire Branch of the Association.)

Derrrium, whenever it arises, is always a matter of serious consideration to the medical practitioner, bnt especially so when it takes place in a disease, or during the progress of a case, in which its presence is unusual, and therefore unlooked for. In such circumstances it is often a subject of great embarrassment, and calls for the most serious reflection on the part of the professional attendant, and requires much experience and practical tact to ascertain its source, probable consequences, and what would be the best method to remove so serious a complication. It is scarcely necessary for me to enumerate the various ordinary sources of delirium, or to observe that the danger accruing from it can only be estimated by the peculiar circumstances of each individual case in which it may happen to occur.

To attain to a satisfactory prognosis, each case must therefore be carefully studied for itself; but it will be necessary to bring to our aid, a tolerably extended view of disease generally, and as great an amount of collateral medical knowledge as may be thought directly or indirectly to bear upon the case specially under treatment.

As in the treatment of all diseases, the subject of diagnosis is of primary importance; it is so in a peculiar sense in the complication here alluded to. It is to be presumed that the original disease upon which delirium has been engrafted, has been correctly understood; in that case it only remains to diagnose the complication, or in other words, to seek for the cause of the delirium; this is sometimes easily discovered, at others it is a matter of great difficulty. We shall be assisted in this inquiry by taking a brief review of delirium as it ordinarily occurs in the routine of medical practice.

The most dangerous cases of delirium, are those in which it is found connected with inflammation of the membranes, or substance of the brain, commonly spoken of as phrenitis; in this disease the delirium is of a violent character, and for the most part comes on suddenly, accompanied with much heat of the surface, flushed countenance, injection of the conjunctiva, with a frequent, strong, and full pulse, and very commonly a red and dry tongue, with a dislike to a strong light and loud sourds. The delirium of typhus fever is generally of a low muttering description, mostly super vening late in the disease, and the history of the case is, in general, sufficiently indicative of its nature. This form of delirium is frequently conjoined with subsultus tendinum, which in the late stages of typhus may be looked upon as a pathognomonic distinction.

As all forms of mental alienation may be said to possess in some degree a generic analogy. Maniacal insanity may sometimes be confounded with true de- 